\title{
THE FRANKE FILTRATION OF THE SPACES OF AUTOMORPHIC FORMS SUPPORTED IN A MAXIMAL PROPER PARABOLIC SUBGROUP
}

\author{
Neven Grbac \\ University of Rijeka, Croatia
}

\begin{abstract}
The Franke filtration is a finite filtration of certain spaces of automorphic forms on the adèlic points of a reductive linear algebraic group defined over a number field whose quotients can be described in terms of parabolically induced representations. Decomposing the space of automorphic forms according to their cuspidal support, the Franke filtration can be made more explicit. This paper describes explicitly the Franke filtration of the spaces of automorphic forms supported in a maximal proper parabolic subgroup, that is, in a cuspidal automorphic representation of its Levi factor. Such explicit description is important for applications to computation of automorphic cohomology, and thus the cohomology of congruence subgroups. As examples, the general linear group and split symplectic and special orthogonal groups are treated.
\end{abstract}

\section{INTRODUCTION}

Let $G$ be a connected reductive linear algebraic group defined over the field $\mathbb{Q}$ of rational numbers. Let $\mathcal{A}$ be the space of automorphic forms on the adèle group $G(\mathbb{A})$, where $\mathbb{A}$ is the ring of adèles of $\mathbb{Q}$. We refer the reader to [3], or Section 2 below, for a precise definition of automorphic forms. The space $\mathcal{A}$ is a $\left(\mathfrak{g}, K_{\infty} ; G\left(\mathbb{A}_{f}\right)\right)$-module, where $\mathfrak{g}$ is the Lie algebra of $G(\mathbb{R}), K_{\infty}$ a fixed maximal compact subgroup of $G(\mathbb{R})$, and $\mathbb{A}_{f}$ the ring of finite adèles of $\mathbb{Q}$.

Let $\mathcal{Z}\left(\mathfrak{g}_{\mathbb{C}}\right)$ be the center of the universal enveloping algebra of the complexification of $\mathfrak{g}$. By definition, any automorphic form on $G(\mathbb{A})$ is annihilated by an ideal in $\mathcal{Z}\left(\mathfrak{g}_{\mathbb{C}}\right)$ of finite codimension. We fix such an ideal $\mathcal{J}$, and

2010 Mathematics Subject Classification. 22E55, 11F70.

Key words and phrases. Automorphic forms, Franke filtration, Eisenstein series. 
define $\mathcal{A}_{\mathcal{J}}$ to be the subspace of $\mathcal{A}$ consisting of automorphic forms that are annihilated by a power of $\mathcal{J}$.

The space of automorphic forms on $G(\mathbb{A})$ can be decomposed, as a $\left(\mathfrak{g}, K_{\infty} ; G\left(\mathbb{A}_{f}\right)\right)$-module, along the cuspidal support (cf. $\left.[8,15,18]\right)$. The summands are indexed by associate classes of cuspidal automorphic representations of the Levi factors of parabolic $\mathbb{Q}$-subgroups belonging to an associate class of parabolic $\mathbb{Q}$-subgroups of $G$. Given an associate class $\{P\}$ of parabolic $\mathbb{Q}$-subgroups of $G$, represented by $P$, and an associate class $\varphi(\pi)$ of cuspidal automorphic representations of the Levi factors of parabolic $\mathbb{Q}$-subgroups in $\{P\}$, represented by a cuspidal automorphic representation $\pi$ of the Levi factor $L_{P}$ of $P$, we denote by $\mathcal{A}_{\mathcal{J},\{P\}, \varphi(\pi)}$ the corresponding summand in the decomposition of $\mathcal{A}_{\mathcal{J}}$. This is the notation as in [8], and we refer to Section 1 of [8], or Section 2 below, for definitions. We say that the automorphic forms in $\mathcal{A}_{\mathcal{J},\{P\}, \varphi(\pi)}$ have cuspidal support in the associate class $\varphi(\pi)$.

Franke defines, in Section 6 of his paper [7], certain filtration of the spaces $\mathcal{A}_{\mathcal{J},\{P\}}$ of automorphic forms on $G(\mathbb{A})$. The space $\mathcal{A}_{\mathcal{J},\{P\}}$ is the sum of spaces $\mathcal{A}_{\mathcal{J},\{P\}, \varphi(\pi)}$ mentioned above over all appropriate $\varphi(\pi)$. The quotients of the filtration are described in terms of induced representations. The Franke filtration is refined to individual summands $\mathcal{A}_{\mathcal{J},\{P\}, \varphi(\pi)}$ in Section 1 of [8]. However, the quotients are described as spanned by the main values of derivatives of appropriate Eisenstein series, and not in terms of induced representations.

The main purpose of this paper is to prove that the Franke filtration of the space $\mathcal{A}_{\mathcal{J},\{P\}, \varphi(\pi)}$ of automorphic forms on $G(\mathbb{A})$, where $P$ is a maximal proper parabolic $\mathbb{Q}$-subgroup of $G$ and $\pi$ a cuspidal automorphic representation of the Levi factor $L_{P}(\mathbb{A})$, has at most two non-trivial steps, and to describe the filtration quotients explicitly in terms of induced representations. This is the subject of our main Theorem 3.1.

More precisely, we prove that there are two non-trivial filtration steps if and only if the Eisenstein series attached to $\pi$ has a pole at the appropriate value $s>0$ of its complex parameter. In this case, the lower filtration step is the $\left(\mathfrak{g}, K_{\infty} ; G\left(\mathbb{A}_{f}\right)\right)$-submodule $\mathcal{L}_{\mathcal{J},\{P\}, \varphi(\pi)}$ spanned by the residues of these Eisenstein series, which are square-integrable automorphic forms. We also describe the quotient $\mathcal{A}_{\mathcal{J},\{P\}, \varphi(\pi)} / \mathcal{L}_{\mathcal{J},\{P\}, \varphi(\pi)}$ explicitly in terms of induced representations. In the other case, that is, if there is only one step in the Franke filtration, we describe the space $\mathcal{A}_{\mathcal{J},\{P\}, \varphi(\pi)}$ itself in terms of induced representations.

Some of these facts were already used, without proof and in a less explicit form, in several papers dealing with the case of a maximal proper parabolic $\mathbb{Q}$-subgroup of a particular classical group $G$. The case $G=G L_{n}$ is considered in [8, Section 5], the case $G=S p_{2 n}$ in [12], and the case $G=G L_{2}$ over a division algebra in [13]. In all these papers, the motivation for studying the Franke filtration is to determine the contribution of residues of Eisenstein 
series supported in a maximal proper parabolic $\mathbb{Q}$-subgroup to the Eisenstein cohomology of the considered group $G$ (with coefficients given by a finitedimensional algebraic representation of $G$ ). Therefore, with the exception of [8], these papers are only focused on the description of the lowest filtration step $\mathcal{L}_{\mathcal{J},\{P\}, \varphi(\pi)}$, which is spanned by the (square-integrable) residues of the Eisenstein series. Having in mind the application to Eisenstein cohomology, these papers also assume that the ideal $\mathcal{J}$ is the annihilator of a finitedimensional representation of $G(\mathbb{C})$. This assumption is quite simplifying, since then only the behavior of the Eisenstein series at certain "not too singular" values of its complex parameter is required.

In the corollaries to the main Theorem 3.1, we consider the case of $\mathbb{Q}$ split classical groups in more detail. Some of these were already considered in the aforementioned papers. However, we consider here arbitrary ideal $\mathcal{J}$, and describe all the filtration quotients explicitly in terms of induced representations. Corollary 4.1 treats the case of the general linear group, and Corollary 4.2 the case of $\mathbb{Q}$-split symplectic and special orthogonal groups for the so-called Siegel maximal proper parabolic $\mathbb{Q}$-subgroup. For these groups the required analytic behavior of the Eisenstein series is known (assuming in Corollary 4.2 Arthur's description of the discrete spectrum).

The importance of having an explicit description of the Franke filtration can be seen in [11]. There, the full Eisenstein cohomology of the $k$-split symplectic group $G=S p_{4}$ of $k$-rank 2 over a totally real algebraic number field $k$ is computed using the explicit description of the filtration quotients in terms of induced representations. However, only the ideals $\mathcal{J}$ that annihilate a finite-dimensional representation of $G(\mathbb{C})$ were considered, as only such ideals matter for cohomology.

The paper is organized as follows. In Section 1 we give the preliminaries and fix the notation regarding the structure of a connected reductive algebraic group defined over $\mathbb{Q}$. The spaces of automorphic forms and their decomposition, as well as the theory of Eisenstein series, are reviewed in Section 2. Section 3 contains the main Theorem 3.1 of the paper, that is, the description of the Franke filtration of the spaces of automorphic forms supported in a maximal proper parabolic $\mathbb{Q}$-subgroup. Finally, in Section 4, as corollaries to Theorem 3.1, we make the description even more precise for $\mathbb{Q}$-split classical groups.

\section{PRELIMINARIES AND NOTATION}

Let $\mathbb{Q}, \mathbb{R}$, and $\mathbb{C}$ denote the field of rational, real, and complex numbers, respectively. Let $\mathbb{Z}$ be the ring of rational integers. For a place $v$ of $\mathbb{Q}$, let $\mathbb{Q} v$ be the completion of $\mathbb{Q}$ at $v$. For the infinite place $v=\infty$, we have $\mathbb{Q}_{v} \cong \mathbb{R}$. Let $\mathbb{A}$ be the ring of adèles of $\mathbb{Q}$, and $\mathbb{A}_{f}$ the ring of finite adèles. 
Let $G$ be a connected reductive linear algebraic group defined over $\mathbb{Q}$. We fix, once for all, a minimal parabolic $\mathbb{Q}$-subgroup $P_{0}$ of $G$. Let $P_{0}=L_{0} N_{0}$ be its Levi decomposition, where $L_{0}$ is the Levi factor and $N_{0}$ the unipotent radical. A parabolic $\mathbb{Q}$-subgroup $P$ of $G$ is called standard if it contains $P_{0}$. We have a Levi decomposition $P=L_{P} N_{P}$, where $L_{P} \supset L_{0}$ is the Levi factor, and $N_{P} \subset N_{0}$ the unipotent radical. Note that $G$ is a parabolic $\mathbb{Q}$-subgroup of itself. We say that a parabolic $\mathbb{Q}$-subgroup is proper if it is not $G$.

Let $P$ be a standard parabolic $\mathbb{Q}$-subgroup of $G$. We denote by $X^{*}\left(L_{P}\right)$ the $\mathbb{Z}$-module of $\mathbb{Q}$-rational characters of its Levi factor $L_{P}$. Let

$$
\check{\mathfrak{a}}_{P}=X^{*}\left(L_{P}\right) \otimes_{\mathbb{Z}} \mathbb{R},
$$

and $\check{\mathfrak{a}}_{P, \mathbb{C}}$ its complexification. In the case $P=P_{0}$, we write $\check{\mathfrak{a}}_{0}$ and $\check{\mathfrak{a}}_{0, \mathbb{C}}$ instead of $\check{\mathfrak{a}}_{P_{0}}$ and $\check{\mathfrak{a}}_{P_{0}, \mathbb{C}}$.

Let $A_{P}$ be the maximal $\mathbb{Q}$-split torus in the center of the Levi factor $L_{P}$, and $X^{*}\left(A_{P}\right)$ the $\mathbb{Z}$-module of $\mathbb{Q}$-rational characters of $A_{P}$. We write $A_{0}$ for $A_{P_{0}}$. The restriction of characters from $L_{P}$ to $A_{P}$ defines an inclusion of $X^{*}\left(L_{P}\right)$ into $X^{*}\left(A_{P}\right)$ as a subgroup of finite index. Hence,

$$
\check{\mathfrak{a}}_{P} \cong X^{*}\left(A_{P}\right) \otimes_{\mathbb{Z}} \mathbb{R}
$$

and the same for $\check{\mathfrak{a}}_{P, \mathbb{C}}$.

Let $P^{\prime}$ be another parabolic $\mathbb{Q}$-subgroup such that $P \subset P^{\prime}$. Then $L_{P} \subset$ $L_{P^{\prime}}$, and the restriction of $\mathbb{Q}$-rational characters from $L_{P^{\prime}}$ to $L_{P}$ defines an injective map from $\check{\mathfrak{a}}_{P^{\prime}}$ into $\check{\mathfrak{a}}_{P}$. We identify $\check{\mathfrak{a}}_{P^{\prime}}$ with a subspace of $\check{\mathfrak{a}}_{P}$ via this map. On the other hand, we also have $A_{P} \supset A_{P^{\prime}}$. Hence, the restriction of characters from $A_{P}$ to $A_{P^{\prime}}$ gives rise to a surjective map in the opposite direction, that is, from $\check{\mathfrak{a}}_{P}$ onto $\check{\mathfrak{a}}_{P^{\prime}}$. This map is, in fact, a projection onto $\check{\mathfrak{a}}_{P^{\prime}}$. Let $\check{\mathfrak{a}}_{P}^{P^{\prime}}$ denote its kernel. It is generated by the characters in $X^{*}\left(L_{P}\right)$ that are trivial on $A_{P^{\prime}}$. Then, we have a direct sum decomposition

$$
\check{\mathfrak{a}}_{P} \cong \check{\mathfrak{a}}_{P^{\prime}} \oplus \check{\mathfrak{a}}_{P}^{P^{\prime}},
$$

and the same for $\check{\mathfrak{a}}_{P, \mathbb{C}}$. In particular, if $P^{\prime}=G$ in the above discussion, then

$$
\check{\mathfrak{a}}_{P} \cong \check{\mathfrak{a}}_{G} \oplus \check{\mathfrak{a}}_{P}^{G} \text {. }
$$

However, if $G$ is semi-simple, the maximal $\mathbb{Q}$-split torus $A_{G}$ in the center of $G$ is trivial, and thus, $\check{\mathfrak{a}}_{P}$ coincides with $\check{\mathfrak{a}}_{P}^{G}$.

Let $\Psi=\Psi\left(G, A_{0}\right)$ denote the set of roots of $G$ with respect to $A_{0}$. It is a root system (cf. [5]), in general non-reduced. Let $W$ be the Weyl group of $G$ with respect to $A_{0}$, that is, the Weyl group of the root system $\Psi$. The fixed choice of $P_{0}$ defines the set $\Psi^{+}$of positive roots, and the set $\Delta$ of simple roots, in $\Psi$.

Observe that $P_{0} \cap L_{P}$ is a minimal parabolic $\mathbb{Q}$-subgroup of the Levi factor $L_{P}$ of a standard parabolic $\mathbb{Q}$-subgroup $P$ of $G$. Let $\Psi_{L_{P}}=\Psi\left(L_{P}, A_{0}\right)$ be the set of roots of $L_{P}$ with respect to $A_{0}$. Then $\Psi_{L_{P}}$ is a subset of $\Psi$, and we define the set $\Psi_{L_{P}}^{+}=\Psi_{L_{P}} \cap \Psi^{+}$of positive roots in $\Psi_{L_{P}}$, and the set 
$\Delta_{L_{P}}=\Psi_{L_{P}} \cap \Delta$ of simple roots in $\Psi_{L_{P}}$. This choice $\Psi_{L_{P}}^{+}$of positive roots defines the open positive Weyl chamber in $\check{\mathfrak{a}}_{P}^{G}$, which we denote $\check{\mathfrak{a}}_{P}^{G,+}$. Let $\rho_{P}$ denote the half-sum of positive roots in $\Psi^{+} \backslash \Psi_{L_{P}}$, that is, positive roots of $G$ that are not the roots of $L_{P}$. Let $W_{L_{P}}$ be the Weyl group of $L_{P}$ with respect to $A_{0}$. It is a subgroup of $W$.

The conjugacy classes of parabolic $\mathbb{Q}$-subgroups of $G$ are in one-to-one correspondence with standard parabolic $\mathbb{Q}$-subgroups, that is, every conjugacy class has a unique standard parabolic $\mathbb{Q}$-subgroup as a representative. Two parabolic $\mathbb{Q}$-subgroups are associate if their Levi factors are conjugate. Clearly, an associate class of parabolic $\mathbb{Q}$-subgroups is a union of conjugacy classes. We denote by $\mathcal{C}$ the set of associate classes of parabolic $\mathbb{Q}$-subgroups of $G$, and by $\{P\} \in \mathcal{C}$ the associate class represented by a parabolic $\mathbb{Q}$-subgroup $P$ of $G$.

Consider the associate class $\{P\} \in \mathcal{C}$ represented by a standard parabolic $\mathbb{Q}$-subgroup $P$ of $G$. Let $W\left(L_{P}\right)$ denote the set of minimal coset representatives for left cosets of $W_{L_{P}}$ in $W$ such that $w L_{P} w^{-1}$ is again a Levi subgroup of a standard parabolic $\mathbb{Q}$-subgroup of $G$. By minimal coset representative we mean the element $w$ of minimal length in its coset $w W_{L_{P}}$. Since $L_{P}$ is obviously stable under conjugation by $W_{L_{P}}$, considering $w L_{P} w^{-1}$ for all $w \in W\left(L_{P}\right)$ gives the Levi factors of all standard parabolic $\mathbb{Q}$-subgroups in the associate class $\{P\}$. For $w \in W\left(L_{P}\right)$, we denote by $P^{w} \in\{P\}$ the standard parabolic $\mathbb{Q}$-subgroup with the Levi factor $w L_{P} w^{-1}$.

We fix, once for all, a maximal compact subgroup $K$ of $G(\mathbb{A})$, which is in good position with respect to $P_{0}$ (as in [18, Sect. I.1.4]). We may and will assume that $K=\prod_{v} K_{v}$, where $K_{v}$ is a fixed maximal compact subgroup of $G\left(\mathbb{Q}_{v}\right)$ for all places $v$ of $\mathbb{Q}$, and $K_{v}$ is hyperspecial for almost all $v$. In particular, $K_{\infty}$ is a maximal compact subgroup of $G(\mathbb{R})$.

Given a standard parabolic $\mathbb{Q}$-subgroup $P$ of $G$, let $X_{*}\left(L_{P}\right)$ be the $\mathbb{Z}$ module of $\mathbb{Q}$-rational cocharacters of the Levi factor $L_{P}$. Let

$$
\mathfrak{a}_{P}=X_{*}\left(L_{P}\right) \otimes_{\mathbb{Z}} \mathbb{R},
$$

and $\mathfrak{a}_{P, \mathbb{C}}$ its complexification. It is dual to the space $\check{\mathfrak{a}}_{P}$, and we denote by $\langle$,$\rangle the standard pairing. We now define the standard height function$ $H_{P}: G(\mathbb{A}) \rightarrow \mathfrak{a}_{P}$. For $l \in L_{P}(\mathbb{A})$ it is defined by the condition

$$
\exp \left\langle\chi, H_{P}(l)\right\rangle=|\chi(l)|
$$

for any character $\chi \in X^{*}\left(L_{P}\right)$, where $|\cdot|$ on the right-hand side is the adèlic absolute value. For $g \in G(\mathbb{A})$, we write $g=\operatorname{lnk}$ according to the Iwasawa decomposition, where $l \in L_{P}(\mathbb{A}), n \in N_{P}(\mathbb{A})$, and $k \in K$. Then, we define $H_{P}(g)=H_{P}(l)$. This is independent on the Iwasawa decomposition of $g$.

Let $\mathfrak{g}$ be the Lie algebra of $G(\mathbb{R})$, and $\mathfrak{g}_{\mathbb{C}}$ its complexification. We denote by $\mathcal{U}\left(\mathfrak{g}_{\mathbb{C}}\right)$ the universal enveloping algebra of $\mathfrak{g}_{\mathbb{C}}$, and by $\mathcal{Z}\left(\mathfrak{g}_{\mathbb{C}}\right)$ its center. 


\section{SPACES OF AUTOMORPHIC FORMS AND EISENSTEIN SERIES}

A smooth function $f: G(\mathbb{A}) \rightarrow \mathbb{C}$ is an automorphic form on $G(\mathbb{A})$ (cf. [3]) if it is

- left $G(\mathbb{Q})$ and $A_{G}(\mathbb{R})^{\circ}$-invariant, where $A_{G}(\mathbb{R})^{\circ}$ is the connected component of $A_{G}(\mathbb{R})$,

- $\mathcal{Z}\left(\mathfrak{g}_{\mathbb{C}}\right)$-finite, that is, annihilated by an ideal in $\mathcal{Z}\left(\mathfrak{g}_{\mathbb{C}}\right)$ of finite codimension,

- $K$-finite, that is, the span of all $f_{k}$ with $k \in K$, where $f_{k}(g)=f(g k)$, is finite-dimensional,

- of uniform moderate growth, that is, there is $r>0$ with the property that for every $D \in \mathcal{U}\left(\mathfrak{g}_{\mathbb{C}}\right)$ there is $C_{D}>0$ such that $|D f(g)| \leq C_{D}\|g\|^{r}$ for all $g \in G(\mathbb{A})$, where $\|\cdot\|$ denotes a norm on $G(\mathbb{A})$ as in $[3$, page 195].

We denote by $\mathcal{A}$ the space of all automorphic forms on $G(\mathbb{A})$. It is a $\left(\mathfrak{g}, K_{\infty} ; G\left(\mathbb{A}_{f}\right)\right)$-module.

For an ideal $\mathcal{J}$ in $\mathcal{Z}\left(\mathfrak{g}_{\mathbb{C}}\right)$ of finite codimension, let $\mathcal{A}_{\mathcal{J}}$ be the $\left(\mathfrak{g}, K_{\infty}\right.$; $G\left(\mathbb{A}_{f}\right)$ )-submodule of $\mathcal{A}$ consisting of automorphic forms annihilated by a power of $\mathcal{J}$. The $\mathcal{Z}\left(\mathfrak{g}_{\mathbb{C}}\right)$-finiteness implies that every automorphic form belongs to $\mathcal{A}_{\mathcal{J}}$ for some ideal $\mathcal{J}$.

For an automorphic form $f$ on $G(\mathbb{A})$, the constant term along a parabolic $\mathbb{Q}$-subgroup $P$ of $G$ is denoted by $f_{P}$. It is defined for $g \in G(\mathbb{A})$ as

$$
f_{P}(g)=\int_{N_{P}(\mathbb{Q}) \backslash N_{P}(\mathbb{A})} f(n g) d n,
$$

where $N_{P}$ is the unipotent radical of $P$, and $d n$ is the Haar measure on the unipotent radical fixed as in $\left[18\right.$, Sect. I.1.13]. Note that if $f_{P}=0$ for all parabolic $\mathbb{Q}$-subgroups $P$ of $G$, then $f=0$. If $f_{P}=0$ for all proper parabolic $\mathbb{Q}$-subgroups $P$ of $G$, then, by definition, $f$ is a cuspidal automorphic form on $G(\mathbb{A})$.

An automorphic form $f$ on $G(\mathbb{A})$ is negligible along a parabolic $\mathbb{Q}$ subgroup $P$, if for all $g \in G(\mathbb{A})$ the function on the Levi factor $L_{P}(\mathbb{A})$, given by the assignment

$$
l \mapsto f_{P}(l g),
$$

is orthogonal to the space of cuspidal automorphic forms on $L_{P}(\mathbb{A})$. Given an associate class $\{P\} \in \mathcal{C}$ of parabolic $\mathbb{Q}$-subgroups of $G$, the space $\mathcal{A}_{\mathcal{J},\{P\}}$ is defined as the $\left(\mathfrak{g}, K_{\infty} ; G\left(\mathbb{A}_{f}\right)\right)$-submodule of $\mathcal{A}_{\mathcal{J}}$ consisting of automorphic forms in $\mathcal{A}_{\mathcal{J}}$ such that their constant term is negligible along all parabolic $\mathbb{Q}$-subgroups outside the associate class $\{P\}$. We say that the automorphic forms in $\mathcal{A}_{\mathcal{J},\{P\}}$ are supported in the associate class $\{P\}$. There is a direct sum decomposition

$$
\mathcal{A}_{\mathcal{J}}=\bigoplus_{\{P\} \in \mathcal{C}} \mathcal{A}_{\mathcal{J},\{P\}}
$$


first proved in [14]. Note that the summand $\mathcal{A}_{\mathcal{J},\{G\}}$, indexed by a singleton $\{G\}$, is the $\left(\mathfrak{g}, K_{\infty} ; G\left(\mathbb{A}_{f}\right)\right)$-module of all cuspidal automorphic forms on $G(\mathbb{A})$ annihilated by a power of $\mathcal{J}$.

Let $\pi$ be a cuspidal automorphic representation of $L_{P}(\mathbb{A})$, that is, an irreducible submodule of the $\left(\mathfrak{l}_{P}, K_{\infty} \cap L_{P}(\mathbb{A}) ; L_{P}\left(\mathbb{A}_{f}\right)\right)$-module of cuspidal automorphic forms on $L_{P}(\mathbb{A})$. We are not assuming that $\pi$ is unitary. However, there is a unitary cuspidal automorphic representation $\pi_{0}$, and an element $\lambda_{0} \in \check{\mathfrak{a}}_{P}^{G}$, such that

$$
\pi \cong \pi_{0} \otimes \exp \left\langle\lambda_{0}, H_{P}(\cdot)\right\rangle .
$$

Thus, the space of $\pi_{0}$ is the space of smooth $K$-finite vectors in an irreducible subrepresentation of the space $L_{\text {cusp }}^{2}\left(L_{P}(\mathbb{A})\right)$ of left $A_{P}(\mathbb{R})^{\circ}$-invariant cuspidal $L^{2}$ automorphic forms on $L_{P}(\mathbb{A})$.

Given a unitary cuspidal automorphic representation $\pi_{0}$ of $L_{P}(\mathbb{A})$, we define the Eisenstein series attached to $\pi_{0}$ as follows. Let $V_{\pi_{0}}$ be the space of smooth $K$-finite functions in the $\pi_{0}$-isotypic component of $L_{\text {cusp }}^{2}\left(L_{P}(\mathbb{A})\right)$. Let $W_{\pi_{0}}$ denote the space of smooth $K$-finite functions

$$
f: L_{P}(\mathbb{Q}) N_{P}(\mathbb{A}) A_{P}(\mathbb{R})^{\circ} \backslash G(\mathbb{A}) \rightarrow \mathbb{C}
$$

such that for all $g \in G(\mathbb{A})$ the function on $L_{P}(A)$, given by the assignment $l \mapsto f(l g)$ for $l \in L_{P}(\mathbb{A})$, belongs to $V_{\pi_{0}}$. For $\lambda \in \check{\mathfrak{a}}_{P, \mathbb{C}}^{G}$ and $f \in W_{\pi_{0}}$, let

$$
f_{\lambda}(g)=f(g) \exp \left\langle\lambda+\rho_{P}, H_{P}(g)\right\rangle,
$$

and $W_{\pi_{0}, \lambda}$ the space of all such $f_{\lambda}$. Then, the space $W_{\pi_{0}, \lambda}$ with the $\left(\mathfrak{g}, K_{\infty} ; G\left(\mathbb{A}_{f}\right)\right)$-module structure given by right translations is naturally isomorphic to the parabolically induced representation

$$
I\left(\lambda, V_{\pi_{0}}\right)=\operatorname{Ind}_{P(\mathbb{A})}^{G(\mathbb{A})}\left(V_{\pi_{0}} \otimes \exp \left\langle\lambda, H_{P}(\cdot)\right\rangle\right),
$$

where the induction is normalized. However, we may realize this induced representation on the space $W_{\pi_{0}}$ as well, with the action depending on $\lambda$. Now the Eisenstein series attached to $f \in W_{\pi_{0}}$ and a complex parameter $\lambda \in \check{\mathfrak{a}}_{P, \mathbb{C}}^{G}$ is defined, at least formally, as

$$
E(f, \lambda)(g)=\sum_{\gamma \in P(\mathbb{Q}) \backslash G(\mathbb{Q})} f_{\lambda}(\gamma g)=\sum_{\gamma \in P(\mathbb{Q}) \backslash G(\mathbb{Q})} f(\gamma g) \exp \left\langle\lambda+\rho_{P}, H_{P}(\gamma g)\right\rangle .
$$

The defining series of the Eisenstein series converges absolutely for the real part of $\lambda$ deep enough inside the positive Weyl chamber $\check{\mathfrak{a}}_{P, \mathbb{C}}^{G,+}$, and has a meromorphic continuation to all of $\check{\mathfrak{a}}_{P, \mathbb{C}}^{G}$ (for the proof of these facts see $[15$, Sect. 7] or [18, Sect. II.1.5 and Chap. IV]).

Let $\{P\} \in \mathcal{C}$ be an associate class of parabolic $\mathbb{Q}$-subgroups of $G$, and let $\pi$ be a cuspidal automorphic representation of the Levi factor $L_{P}(\mathbb{A})$. The associate class $\varphi(\pi)$, represented by $\pi$, of cuspidal automorphic 
representations of the Levi factors of parabolic $\mathbb{Q}$-subgroups in $\{P\}$ is a collection of finite sets

$$
\varphi(\pi)=\left\{\varphi_{Q}(\pi)\right\}_{Q \in\{P\}},
$$

indexed by parabolic $\mathbb{Q}$-subgroups $Q$ in the associate class $\{P\}$. The set $\varphi_{Q}(\pi)$ consists of cuspidal automorphic representations of $L_{Q}(\mathbb{A})$, and is defined as

$$
\varphi_{Q}(\pi)=\left\{\pi^{w}: w \in W\left(L_{P}\right) \text { such that } L_{Q}=w L_{P} w^{-1}\right\},
$$

where $\pi^{w}(l)=\pi\left(w^{-1} l w\right)$, for all $l \in L_{Q}(\mathbb{A})$. Since $Q$ is associate to $P$, the set of $w \in W\left(L_{P}\right)$ such that $L_{Q}=w L_{P} w^{-1}$ is not empty, and thus, $\varphi_{Q}(\pi)$ is not empty. We say that $\pi$ and $\pi^{w}$ are associate cuspidal automorphic representations. It is clear that being associate is an equivalence relation on the set of all cuspidal automorphic representations of the Levi factors of parabolic $\mathbb{Q}$-subgroups of $G$.

In order to have a non-trivial contribution of the associate class $\varphi(\pi)$ to the space $\mathcal{A}_{\mathcal{J}}$, it is necessary to impose certain compatibility condition on $\pi$ with respect to $\mathcal{J}$. Let $\chi$ be the infinitesimal character of the Archimedean component of $\pi$. We say that the associate class $\varphi(\pi)$ is compatible with $\mathcal{J}$ if the Weyl group orbit of the infinitesimal character $\chi$ is annihilated by the ideal $\mathcal{J}$. Here $\mathcal{J}$ is viewed, via the Harish-Chandra isomorphism, as an ideal of the algebra $S\left(\check{\mathfrak{a}}_{0}\right)^{W}$ of the Weyl group invariant elements in the symmetric algebra $S\left(\check{\mathfrak{a}}_{0}\right)$ of $\check{\mathfrak{a}}_{0}$. Let $\Phi_{\mathcal{J},\{P\}}$ denote the set of all associate classes $\varphi(\pi)$ that are compatible with $\mathcal{J}$.

By replacing $\pi$ with another cuspidal automorphic representation in the same associate class, we may and will assume that $P$ is a standard parabolic $\mathbb{Q}$-subgroup, and the corresponding $\lambda_{0} \in \check{\mathfrak{a}}_{P}^{G}$, defined as above, belongs to the closure of the positive Weyl chamber $\check{\mathfrak{a}}_{P}^{G,+}$. The poles inside $\check{\mathfrak{a}}_{P}^{G,+}$ of the Eisenstein series attached to functions $f \in W_{\pi_{0}}$ all lie along the locally finite family of singular hyperplanes (see [18, Sect. IV.1]). Therefore, there is a polynomial $p(\lambda)$ such that

$$
p(\lambda) E(f, \lambda)(g)
$$

is holomorphic in the neighborhood of $\lambda_{0}$.

Assume that $\varphi(\pi) \in \Phi_{\mathcal{J},\{P\}}$. The space $\mathcal{A}_{\mathcal{J},\{P\}, \varphi(\pi)}$ of automorphic forms with the cuspidal support in the associate class $\varphi(\pi)$, is defined in Section 1.3 of [8]. It is the linear span of the coefficients in the Taylor expansion of $p(\lambda) E(f, \lambda)(g)$ around $\lambda=\lambda_{0}$ in some choice of Cartesian coordinates. This definition is independent on the choice of the polynomial $p(\lambda)$, and the representatives for $\{P\}$ and $\varphi(\pi)$. It is proved in [8, Sect. 1.4], and also in $[18$, Sect. III.2] following a different approach, that there is a direct sum decomposition along cuspidal support

$$
\mathcal{A}_{\mathcal{J}} \cong \bigoplus_{\{P\} \in \mathcal{C}} \bigoplus_{\varphi(\pi) \in \Phi_{\mathcal{J},\{P\}}} \mathcal{A}_{\mathcal{J},\{P\}, \varphi(\pi)}
$$

of $\left(\mathfrak{g}, K_{\infty} ; G\left(\mathbb{A}_{f}\right)\right)$-modules. 
In Section 6 of [7], Franke defines a filtration of the $\left(\mathfrak{g}, K_{\infty} ; G\left(\mathbb{A}_{f}\right)\right)$ module $\mathcal{A}_{\mathcal{J},\{P\}}$. However, intersecting with $\mathcal{A}_{\mathcal{J},\{P\}, \varphi(\pi)}$, one obtains the Franke filtration of the $\left(\mathfrak{g}, K_{\infty} ; G\left(\mathbb{A}_{f}\right)\right)$-module $\mathcal{A}_{\mathcal{J},\{P\}, \varphi(\pi)}$. It is a descending filtration

$$
\cdots \supseteq \mathcal{A}_{\mathcal{J},\{P\}, \varphi(\pi)}^{i} \supseteq \mathcal{A}_{\mathcal{J},\{P\}, \varphi(\pi)}^{i+1} \supseteq \ldots,
$$

indexed by $i \in \mathbb{Z}$, in which only finitely many steps are non-trivial. We give more details regarding the Franke filtration, at least for a maximal proper parabolic $\mathbb{Q}$-subgroup $P$, in the proof of Theorem 3.1 below.

\section{The Franke filtration of $\mathcal{A}_{\mathcal{J},\{P\}, \varphi(\pi)}$ FOR $P$ MaXimal Proper PARABOLIC $\mathbb{Q}$-SUBGROUP}

In this section, $P$ is a standard maximal proper parabolic $\mathbb{Q}$-subgroup of $G$. Since $P$ is maximal proper, there is a unique simple root $\alpha \in \Delta$, which is not a root in $\Psi_{L_{P}}$, and the space $\mathfrak{\mathfrak { a }}_{P}^{G}$ is one-dimensional. As in [20], we fix

$$
\tilde{\alpha}=\left\langle\rho_{P}, \alpha^{\vee}\right\rangle^{-1} \rho_{P}
$$

to be the basis of $\check{\mathfrak{a}}_{P}^{G}$, where $\alpha^{\vee}$ is the dual root of $\alpha$. Thus, we may write an element $\lambda \in \check{\mathfrak{a}}_{P, \mathbb{C}}^{G}$ as $\lambda=s \tilde{\alpha}$, where $s=\left\langle\lambda, \alpha^{\vee}\right\rangle \in \mathbb{C}$. In this basis, the open positive Weyl chamber in $\check{\mathfrak{a}}_{P, \mathbb{C}}^{G}$ consists of all $s \tilde{\alpha} \in \check{\mathfrak{a}}_{P, \mathbb{C}}^{G}$ such that $\operatorname{Re}(s)>0$.

Let $P=L_{P} N_{P}$ be the Levi decomposition of $P$. There is a unique nontrivial element $w_{0}$ in $W\left(L_{P}\right)$. Recall that a standard parabolic $\mathbb{Q}$-subgroup of $G$ is self-associate if it is the only standard parabolic $\mathbb{Q}$-subgroup in its associate class. In our case, $P$ is self-associate if and only if $P^{w_{0}}=P$. Otherwise, that is, if $P$ is not self-associate, there are two standard parabolic $\mathbb{Q}$-subgroups in $\{P\}$, namely, $P$ and $P^{w_{0}}$.

Let $\pi$ be a cuspidal automorphic representation of $L_{P}(\mathbb{A})$. Write

$$
\pi \cong \pi_{0} \otimes \exp \left\langle s_{0} \tilde{\alpha}, H_{P}(\cdot)\right\rangle,
$$

as in Section 2, where $\pi_{0}$ is a unitary cuspidal automorphic representation of $L_{P}(\mathbb{A})$, and $s_{0} \tilde{\alpha} \in \check{\mathfrak{a}}_{P}^{G}$, i.e., $s_{0} \in \mathbb{R}$. As explained in Section 2, it is not restrictive to assume that the representative $\pi$ of the associate class $\varphi(\pi)$ is such that $s_{0} \geq 0$. We also assume that the associate class $\varphi(\pi)$ is compatible with $\mathcal{J}$, since otherwise the space $\mathcal{A}_{\mathcal{J},\{P\}, \varphi(\pi)}$ is trivial.

Let $\pi^{w_{0}}$ be the conjugate of $\pi$ by $w_{0}$. It is a cuspidal automorphic representation of $L_{P w_{0}}(\mathbb{A})$. Then

$$
\pi^{w_{0}} \cong \pi_{0}^{w_{0}} \otimes \exp \left\langle-s_{0} \tilde{\alpha}^{w_{0}}, H_{P^{w_{0}}}(\cdot)\right\rangle,
$$

where $\alpha^{w_{0}}$ is the unique simple root in $\Delta$ not being the root of $L_{P} w_{0}$, and $\pi_{0}^{w_{0}}$ is a unitary cuspidal automorphic representation of $L_{P w_{0}}(\mathbb{A})$ conjugate to $\pi_{0}$. Note that $\alpha^{w_{0}}=\alpha$ if $P^{w_{0}}=P$.

Consider the Eisenstein series

$$
E(f, s \tilde{\alpha})(g)
$$


attached to $\pi_{0}$ as in Section 2, where $s \tilde{\alpha} \in \check{\mathfrak{a}}_{P, \mathbb{C}}^{G}$ and $f \in W_{\pi_{0}}$. It has a finite number of simple poles in the real interval

$$
\left\{s \tilde{\alpha} \in \check{\mathfrak{a}}_{P}^{G}: 0<s \leq\left\langle\rho_{P}, \alpha^{\vee}\right\rangle\right\},
$$

and the other possible poles lie in the region $\operatorname{Re}(s)<0$ (cf. [15, Sect. 6] or $[18$, Sect. IV.3]). Let

$$
\mathcal{L}_{\mathcal{J},\{P\}, \varphi(\pi)}=\left\{\operatorname{Res}_{s=s_{0}} E(f, s \tilde{\alpha})(g): f \in W_{\pi_{0}}\right\}
$$

be the space of the residues at $s=s_{0}$ of the Eisenstein series attached to $\pi_{0}$. Then, $\mathcal{L}_{\mathcal{J},\{P\}, \varphi(\pi)}$ is a $\left(\mathfrak{g}, K_{\infty} ; G\left(\mathbb{A}_{f}\right)\right)$-submodule of $\mathcal{A}_{\mathcal{J},\{P\}, \varphi(\pi)}$, and clearly, it is non-trivial if and only if the Eisenstein series $E(f, s \tilde{\alpha})(g)$ has a pole at $s=s_{0}$ for some $f \in W_{\pi_{0}}$. By the Langlands square integrability criterion (cf. [18, Sect. I.4.11]), the automorphic forms in $\mathcal{L}_{\mathcal{J},\{P\}, \varphi(\pi)}$ are square-integrable. Therefore, the space $\mathcal{L}_{\mathcal{J},\{P\}, \varphi(\pi)}$ is the space of smooth $K$-finite vectors of a residual automorphic representation of $G(\mathbb{A})$.

THEOREM 3.1. Let $G$ be a connected reductive linear algebraic group defined over $\mathbb{Q}$. Let $P$ be a standard maximal proper parabolic $\mathbb{Q}$-subgroup of $G$, and $\pi$ a cuspidal automorphic representation of $L_{P}(\mathbb{A})$. The Franke filtration of the $\left(\mathfrak{g}, K_{\infty} ; G\left(\mathbb{A}_{f}\right)\right)$-module $\mathcal{A}_{\mathcal{J},\{P\}, \varphi(\pi)}$ coincides with the filtration

$$
\{0\} \subseteq \mathcal{L}_{\mathcal{J},\{P\}, \varphi(\pi)} \subseteq \mathcal{A}_{\mathcal{J},\{P\}, \varphi(\pi)},
$$

which consists of at most two non-trivial filtration steps.

The lower filtration step $\mathcal{L}_{\mathcal{J},\{P\}, \varphi(\pi)}$ is non-trivial if and only if the Eisenstein series attached to $\pi_{0}$ have a pole at $s=s_{0} \geq 0$. It consists of square-integrable automorphic forms obtained as residues at $s=s_{0}$ of these Eisenstein series.

The quotient $\mathcal{A}_{\mathcal{J},\{P\}, \varphi(\pi)} / \mathcal{L}_{\mathcal{J},\{P\}, \varphi(\pi)}$ is always non-trivial, and isomorphic as a $\left(\mathfrak{g}, K_{\infty} ; G\left(\mathbb{A}_{f}\right)\right)$-module to

$\mathcal{A}_{\mathcal{J},\{P\}, \varphi(\pi)} / \mathcal{L}_{\mathcal{J},\{P\}, \varphi(\pi)} \cong\left\{\begin{array}{l}\left(I\left(0, V_{\pi_{0}}\right) \otimes S\left(\check{\mathfrak{a}}_{P, \mathbb{C}}^{G}\right)\right)^{+}, \quad \text { if }\left\{\begin{array}{c}s_{0}=0 \\ \text { and } P^{w_{0}}=P, \\ \text { and } \pi_{0}^{w_{0}} \cong \pi_{0}\end{array}\right. \\ I\left(s_{0} \tilde{\alpha}, V_{\pi_{0}}\right) \otimes S\left(\check{\mathfrak{a}}_{P, \mathbb{C}}^{G}\right), \quad \text { otherwise, }\end{array}\right.$

where $S\left(\check{\mathfrak{a}}_{P, \mathbb{C}}^{G}\right)$ is the symmetric algebra of $\check{\mathfrak{a}}_{P, \mathbb{C}}^{G}$, and the $\left(\mathfrak{g}, K_{\infty} ; G\left(\mathbb{A}_{f}\right)\right)$ module structure on the right-hand side is given as in [7, page 218]. The space

$$
\left(I\left(0, V_{\pi_{0}}\right) \otimes S\left(\check{\mathfrak{a}}_{P, \mathbb{C}}^{G}\right)\right)^{+}
$$

is the 1-eigenspace of the self-intertwining operator $M\left(w_{0}\right)$ acting on the space $I\left(0, V_{\pi_{0}}\right) \otimes S\left(\check{\mathfrak{a}}_{P, \mathbb{C}}^{G}\right)$ defined in [7, page 234].

Proof. The proof is based on Theorem 14 of [7], in which the filtration quotients for the $\left(\mathfrak{g}, K_{\infty} ; G\left(\mathbb{A}_{f}\right)\right)$-module $\mathcal{A}_{\mathcal{J},\{P\}}$ are described in terms of 
induced representations, combined with the cuspidal support point of view taken in the proof of Theorem 1.4 of [8].

Given $P$ and $\pi$ as in the theorem, let $\mathcal{M}_{\mathcal{J},\{P\}, \varphi(\pi)}$ denote the set of triples $(R, \Pi, \lambda)$, where

- $R$ is a standard parabolic $\mathbb{Q}$-subgroup containing an element of the associate class $\{P\}$,

- $\Pi$ is a discrete spectrum representation of the Levi factor $L_{R}(\mathbb{A})$,

- $\lambda$ is an element of the closure of the positive Weyl chamber $\check{\mathfrak{a}}_{R}^{G,+}$, such that the representation

$$
\Pi \otimes \exp \left\langle\lambda, H_{R}(\cdot)\right\rangle
$$

of $L_{R}(\mathbb{A})$ is supported in the associate class $\varphi(\pi)$, and the Weyl group orbits of its infinitesimal character and the infinitesimal character of $\pi$ coincide; in particular, it is compatible with $\mathcal{J}$.

In our case, since $P$ is maximal, there are not many possibilities for $R$. We have either $R=G$, or $R$ in the associate class $\{P\}$.

For $R=G, \Pi$ is necessarily a residual representation of $G(\mathbb{A})$ supported in $\varphi(\pi)$, and clearly $\lambda=0$. Such $\Pi$ exists if and only if the Eisenstein series attached to $\pi_{0}$ has a pole at $s=s_{0}$. Then, $\Pi$ is isomorphic to the space $\mathcal{L}_{\mathcal{J},\{P\}, \varphi(\pi)}$ spanned by the residues. Hence,

$$
\left(G, \mathcal{L}_{\mathcal{J},\{P\}, \varphi(\pi)}, 0\right) \in \mathcal{M}_{\mathcal{J},\{P\}, \varphi(\pi)},
$$

if and only if $\mathcal{L}_{\mathcal{J},\{P\}, \varphi(\pi)}$ is non-trivial.

For $R$ in the associate class $\{P\}$, there is always the triple

$$
\left(P, V_{\pi_{0}}, s_{0} \tilde{\alpha}\right) \in \mathcal{M}_{\mathcal{J},\{P\}, \varphi(\pi)} .
$$

Another candidate for a triple in $\mathcal{M}_{\mathcal{J},\{P\}, \varphi(\pi)}$ is the conjugate by $w_{0}$ of the previous one, that is,

$$
\left(P^{w_{0}}, V_{\pi_{0}^{w_{0}}},-s_{0} \tilde{\alpha}^{w_{0}}\right) \text {. }
$$

However, if $s_{0}>0$, then $-s_{0} \tilde{\alpha}^{w_{0}}$ is not in the closure of the positive Weyl chamber $\check{\mathfrak{a}}_{P w_{0}}^{G,+}$, so the conjugate triple is never in $\mathcal{M}_{\mathcal{J},\{P\}, \varphi(\pi)}$. Suppose that $s_{0}=0$. If $P^{w_{0}}=P$ and $\pi^{w_{0}} \cong \pi_{0}$, then the conjugate triple coincides with the original one. Otherwise, that is, if $s_{0}=0$, and $P^{w_{0}} \neq P$ or $\pi_{0}^{w_{0}} \not \pi_{0}$, the conjugate triple is another triple in $\mathcal{M}_{\mathcal{J},\{P\}, \varphi(\pi)}$.

We conclude the above discussion by listing the sets $\mathcal{M}_{\mathcal{J},\{P\}, \varphi(\pi)}$ in all possible cases. There are four cases:

CASE $1 \mathcal{L}_{\mathcal{J},\{P\}, \varphi(\pi)} \neq\{0\}$, which implies that $s_{0}>0$, and $P^{w_{0}}=P$ and $\pi_{0}^{w_{0}} \cong \pi_{0}$,

CASE $2 s_{0}>0$ and $\mathcal{L}_{\mathcal{J},\{P\}, \varphi(\pi)}=\{0\}$,

CASE $3 s_{0}=0$ and $P^{w_{0}}=P$ and $\pi^{w_{0}} \cong \pi_{0}$, which implies that $\mathcal{L}_{\mathcal{J},\{P\}, \varphi(\pi)}=\{0\}$,

CASE $4 s_{0}=0$, and $P^{w_{0}} \neq P$ or $\pi^{w_{0}} \neq \pi_{0}$, which implies that $\mathcal{L}_{\mathcal{J},\{P\}, \varphi(\pi)}=\{0\}$. 
Observe that, since the Eisenstein series is holomorphic on the imaginary axis (cf. [18, Sect. IV.1]), we have $s_{0}>0$ in case 1 , and $\mathcal{L}_{\mathcal{J},\{P\}, \varphi(\pi)}=\{0\}$ whenever $s_{0}=0$, that is, in case 3 and case 4. According to [18, Sect. IV.3], if $P^{w_{0}} \neq P$ or $\pi_{0}^{w_{0}} ¥ \pi_{0}$, the Eisenstein series attached to $\pi_{0}$ is holomorphic in $R e(s) \geq 0$. Since in case 1 there is a pole of this Eisenstein series at $s=s_{0}>0$, it follows that $P^{w_{0}}=P$ and $\pi_{0}^{w_{0}} \cong \pi_{0}$. Then

$$
\mathcal{M}_{\mathcal{J},\{P\}, \varphi(\pi)}= \begin{cases}\left\{\left(G, \mathcal{L}_{\mathcal{J},\{P\}, \varphi(\pi)}, 0\right),\left(P, V_{\pi_{0}}, s_{0} \tilde{\alpha}\right)\right\}, & \text { in case } 1 \\ \left\{\left(P, V_{\pi_{0}}, s_{0} \tilde{\alpha}\right)\right\}, & \text { in case } 2 \\ \left\{\left(P, V_{\pi_{0}}, 0\right)\right\}, & \text { in case } 3 \\ \left\{\left(P, V_{\pi_{0}}, 0\right),\left(P^{w_{0}}, V_{\pi_{0}}^{w_{0}}, 0\right)\right\}, & \text { in case } 4\end{cases}
$$

For a pair of triples $(R, \Pi, \lambda)$ and $\left(R^{\prime}, \Pi^{\prime}, \lambda^{\prime}\right)$ in $\mathcal{M}_{\mathcal{J},\{P\}, \varphi(\pi)}$, we define the set of morphisms as the set of all $w \in W\left(L_{R}\right)$ such that $R^{\prime}=R^{w}$, $\Pi^{\prime}=\Pi^{w}$, and $\lambda^{\prime}=\lambda^{w}$. This turns $\mathcal{M}_{\mathcal{J},\{P\}, \varphi(\pi)}$ into a finite category, which is a groupoid, since every morphism is an isomorphism. The assignment

$$
(R, \Pi, \lambda) \mapsto I(\lambda, \Pi) \otimes S\left(\check{\mathfrak{a}}_{R, \mathbb{C}}^{G}\right)
$$

defines a functor, which we denote by $M$, from $\mathcal{M}_{\mathcal{J},\{P\}, \varphi(\pi)}$ to the category of $\left(\mathfrak{g}, K_{\infty} ; G\left(\mathbb{A}_{f}\right)\right)$-modules. This functor maps a morphism $w$ of triples in $\mathcal{M}_{\mathcal{J},\{P\}, \varphi(\pi)}$ to an intertwining operator $M(w)$ attached to $w$ of corresponding $\left(\mathfrak{g}, K_{\infty} ; G\left(\mathbb{A}_{f}\right)\right)$-modules as defined in [7, page 234]. Since we do not need a precise definition, we only remark that the definition of $M(w)$ involves not only the standard intertwining operators acting on induced representations (cf. [18, Sect. II.1.6]), but also their derivatives.

In our situation, the category $\mathcal{M}_{\mathcal{J},\{P\}, \varphi(\pi)}$ is discrete in case 1 and case 2 , that is, the only morphisms are identities. This is due to the fact that $s_{0}>0$, so that $-s_{0} \tilde{\alpha}^{w_{0}}$ is no longer in the closure of the positive Weyl chamber. In case 3 , we have a single object in $\mathcal{M}_{\mathcal{J},\{P\}, \varphi(\pi)}$, which has two morphisms: the identity and $w_{0}$. In case 4 , we have two objects, and a single morphism between any pair of objects: the identities on each of the two objects, and the morphisms $w_{0}$ and $w_{0}^{-1}$ between them.

The final ingredient for the filtration is an integer valued function $T_{\mathcal{J},\{P\}, \varphi(\pi)}$. It is defined on the subset $S_{\mathcal{J},\{P\}, \varphi(\pi)}$ of $\check{\mathfrak{a}}_{0}$ consisting of all $\lambda \in \check{\mathfrak{a}}_{R}^{G}$ appearing as the third entry of a triple in $\mathcal{M}_{\mathcal{J},\{P\}, \varphi(\pi)}$, viewed as elements of $\check{\mathfrak{a}}_{0}$ via the embedding of $\check{\mathfrak{a}}_{R}^{G}$ into $\check{\mathfrak{a}}_{0}$. This set is finite in general, because $\lambda$ is required to be compatible with $\mathcal{J}$, meaning that $\lambda$ is among restrictions to $\check{\mathfrak{a}}_{R}^{G}$ of elements in $\check{\mathfrak{a}}_{0}$ annihilated by $\mathcal{J}$. There is only finitely many such restrictions, since the ideal $\mathcal{J}$ of finite codimension in $\mathcal{Z}\left(\mathfrak{g}_{\mathbb{C}}\right)$, viewed via the Harish-Chandra isomorphism as an ideal in $S\left(\check{\mathfrak{a}}_{0}\right)^{W}$, annihilates 
a finite set of Weyl group orbits in $\check{\mathfrak{a}}_{0}$. In our case, we have

$$
S_{\mathcal{J},\{P\}, \varphi(\pi)}= \begin{cases}\left\{0, s_{0} \tilde{\alpha}\right\}, & \text { in case } 1, \\ \left\{s_{0} \tilde{\alpha}\right\}, & \text { in case } 2, \\ \{0\}, & \text { in case } 3 \text { and case } 4,\end{cases}
$$

where $s_{0}>0$. Function $T_{\mathcal{J},\{P\}, \varphi(\pi)}$ is not unique. It is any function defined on the set $S_{\mathcal{J},\{P\}, \varphi(\pi)}$ with values in $\mathbb{Z}$ satisfying the property given in [7, page 233]. For the maximal proper parabolic case, this property is equivalent to

$$
T_{\mathcal{J},\{P\}, \varphi(\pi)}\left(s_{1} \tilde{\alpha}\right)>T_{\mathcal{J},\{P\}, \varphi(\pi)}\left(s_{2} \tilde{\alpha}\right) \quad \text { if } \quad s_{1}<s_{2} .
$$

The filtration is independent on the choice of $T_{\mathcal{J},\{P\}, \varphi(\pi)}$ (up to the numeration of filtration steps). We take $T_{\mathcal{J},\{P\}, \varphi(\pi)}$ defined by

$$
\begin{aligned}
T_{\mathcal{J},\{P\}, \varphi(\pi)}(0) & = \begin{cases}1, & \text { in case } 1, \\
0, & \text { in case } 3 \text { and case } 4,\end{cases} \\
T_{\mathcal{J},\{P\}, \varphi(\pi)}\left(s_{0} \widetilde{\alpha}\right) & =0 \quad \text { for } s_{0}>0 .
\end{aligned}
$$

Next, we invoke Franke's Theorem 14 of [7]. Considering the cuspidal support, it provides a description of the quotients of the Franke filtration

$$
\cdots \supseteq \mathcal{A}_{\mathcal{J},\{P\}, \varphi(\pi)}^{i} \supseteq \mathcal{A}_{\mathcal{J},\{P\}, \varphi(\pi)}^{i+1} \supseteq \cdots
$$

of $\mathcal{A}_{\mathcal{J},\{P\}, \varphi(\pi)}$, indexed by $i \in \mathbb{Z}$. More precisely, the quotients are isomorphic as $\left(\mathfrak{g}, K_{\infty} ; G\left(\mathbb{A}_{f}\right)\right)$-modules to

$$
\begin{aligned}
& \mathcal{A}_{\mathcal{J},\{P\}, \varphi(\pi)}^{i} / \mathcal{A}_{\mathcal{J},\{P\}, \varphi(\pi)}^{i+1} \cong \bigoplus_{k=0} \bigoplus_{\substack{(R, \Pi, \lambda) \in \mathcal{M}_{\mathcal{J},\{P\}, \varphi(\pi)} \\
\operatorname{dim} \check{\mathfrak{a}}_{R}^{G}=k \\
T_{\mathcal{J},\{P\}, \varphi(\pi)}^{G}(\lambda)=i}} M(R, \Pi, \lambda) \\
& \cong \bigoplus_{k=0}^{\operatorname{dim} \check{\mathfrak{a}}_{P}^{G}} \operatorname{colim}_{\substack{(R, \Pi, \lambda) \in \mathcal{M} \\
\operatorname{dim} \check{\mathfrak{a}}_{R}^{G}=k \\
T_{\mathcal{J},\{P\}, \varphi(\pi)}, \varphi(\pi)(\lambda)=i}} I(\lambda, \Pi) \otimes S\left(\check{\mathfrak{a}}_{R, \mathbb{C}}^{G}\right),
\end{aligned}
$$

for all $i \in \mathbb{Z}$, where $M$ is the functor, defined above, from the category $\mathcal{M}_{\mathcal{J},\{P\}, \varphi(\pi)}$ to the category of $\left(\mathfrak{g}, K_{\infty} ; G\left(\mathbb{A}_{f}\right)\right)$-modules, and the colimit is the colimit in the category of $\left(\mathfrak{g}, K_{\infty} ; G\left(\mathbb{A}_{f}\right)\right)$-modules of the functor $M$, restricted to the subcategory of all triples in $\mathcal{M}_{\mathcal{J},\{P\}, \varphi(\pi)}$ with $\operatorname{dim} \check{\mathfrak{a}}_{R}^{G}=k$ (cf. [16, Sect. III.3]). Observe that all but finitely many quotients are trivial, since the range of $T_{\mathcal{J},\{P\}, \varphi(\pi)}$ is finite.

In case 1 this description of the quotients implies that the Franke filtration of $\mathcal{A}_{\mathcal{J},\{P\}, \varphi(\pi)}$ is a two-step filtration

$$
\mathcal{A}_{\mathcal{J},\{P\}, \varphi(\pi)}=\mathcal{A}_{\mathcal{J},\{P\}, \varphi(\pi)}^{0} \supset \mathcal{A}_{\mathcal{J},\{P\}, \varphi(\pi)}^{1} \supset\{0\},
$$


as the range of $T_{\mathcal{J},\{P\}, \varphi(\pi)}$ is $\{0,1\}$. Since the category $\mathcal{M}_{\mathcal{J},\{P\}, \varphi(\pi)}$ is discrete, the colimit is just the direct sum of objects, and we have

$$
\mathcal{A}_{\mathcal{J},\{P\}, \varphi(\pi)}^{1} \cong M\left(G, \mathcal{L}_{\mathcal{J},\{P\}, \varphi(\pi)}, 0\right)=\mathcal{L}_{\mathcal{J},\{P\}, \varphi(\pi)},
$$

which is non-trivial precisely in this case. The quotient is isomorphic to

$$
\begin{aligned}
\mathcal{A}_{\mathcal{J},\{P\}, \varphi(\pi)}^{0} / \mathcal{A}_{\mathcal{J},\{P\}, \varphi(\pi)}^{1} & =\mathcal{A}_{\mathcal{J},\{P\}, \varphi(\pi)} / \mathcal{L}_{\mathcal{J},\{P\}, \varphi(\pi)} \\
& \cong M\left(P, V_{\pi_{0}}, s_{0}\right) \\
& \cong I\left(s_{0} \tilde{\alpha}, V_{\pi_{0}}\right) \otimes S\left(\check{\mathfrak{a}}_{P, \mathbb{C}}^{G}\right)
\end{aligned}
$$

This proves the theorem in case 1 .

In the remaining cases, the Franke filtration is just one-step filtration

$$
\mathcal{A}_{\mathcal{J},\{P\}, \varphi(\pi)}=\mathcal{A}_{\mathcal{J},\{P\}, \varphi(\pi)}^{0} \supset\{0\},
$$

as the range of $T_{\mathcal{J},\{P\}, \varphi(\pi)}$ is $\{0\}$. Note that in all these cases $\mathcal{L}_{\mathcal{J},\{P\}, \varphi(\pi)}$ is trivial. Hence, according to the description of the quotients, we have

$$
\begin{aligned}
& \mathcal{A}_{\mathcal{J},\{P\}, \varphi(\pi)}^{0}=\mathcal{A}_{\mathcal{J},\{P\}, \varphi(\pi)} \\
& \cong \operatorname{colim}_{(R, \Pi, \lambda) \in \mathcal{M}_{\mathcal{J},\{P\}, \varphi(\pi)}} M(R, \Pi, \lambda) \\
& \cong \operatorname{colim}_{(R, \Pi, \lambda) \in \mathcal{M}_{\mathcal{J},\{P\}, \varphi(\pi)}} I(\lambda, \Pi) \otimes S\left(\check{\mathfrak{a}}_{R, \mathbb{C}}^{G}\right) .
\end{aligned}
$$

It remains to determine the colimits.

In case 2 , the category $\mathcal{M}_{\mathcal{J},\{P\}, \varphi(\pi)}$ is discrete and consists of a single object. Hence,

$$
\mathcal{A}_{\mathcal{J},\{P\}, \varphi(\pi)}^{0} \cong I\left(s_{0} \tilde{\alpha}, V_{\pi_{0}}\right) \otimes S\left(\check{\mathfrak{a}}_{P, \mathbb{C}}^{G}\right)
$$

in this case.

In case 3 , there is a single object in the category $\mathcal{M}_{\mathcal{J},\{P\}, \varphi(\pi)}$ with two morphisms: the non-trivial morphism $w_{0}$ and the identity. Hence, the colimit is the coequalizer of the two morphisms. That is, it is the quotient of $M\left(P, V_{\pi_{0}}, 0\right)$ by the $\left(\mathfrak{g}, K_{\infty} ; G\left(\mathbb{A}_{f}\right)\right)$-submodule $W$ consisting of all $x-M\left(w_{0}\right) x$, where $x \in M\left(P, V_{\pi_{0}}, 0\right)$, and $M\left(w_{0}\right)$ is the intertwining operator acting on $M\left(P, V_{\pi_{0}}, 0\right)$. On the other hand, using the functional equation for the standard intertwining operators (cf. [18, Sect. IV.1]), one can show that $M\left(w_{0}\right)^{2}$ is the identity on $I\left(0, V_{\pi_{0}}\right) \otimes S\left(\check{\mathfrak{a}}_{P, \mathbb{C}}^{G}\right)$, so that it decomposes into the direct sum

$$
I\left(0, V_{\pi_{0}}\right) \otimes S\left(\check{\mathfrak{a}}_{P, \mathbb{C}}^{G}\right) \cong\left(I\left(0, V_{\pi_{0}}\right) \otimes S\left(\check{\mathfrak{a}}_{P, \mathbb{C}}^{G}\right)\right)^{+} \oplus\left(I\left(0, V_{\pi_{0}}\right) \otimes S\left(\check{\mathfrak{a}}_{P, \mathbb{C}}^{G}\right)\right)^{-}
$$

where $\left(I\left(0, V_{\pi_{0}}\right) \otimes S\left(\check{\mathfrak{a}}_{P, \mathbb{C}}^{G}\right)\right)^{ \pm}$is the \pm 1 -eigenspace of $M\left(w_{0}\right)$. Observe that $W$ is precisely the -1 -eigenspace. Therefore, the quotient is

$$
\mathcal{A}_{\mathcal{J},\{P\}, \varphi(\pi)}^{0} \cong\left(I\left(0, V_{\pi_{0}}\right) \otimes S\left(\check{\mathfrak{a}}_{P, \mathbb{C}}^{G}\right)\right)^{+}
$$

in this case. 
In case 4 , the category $\mathcal{M}_{\mathcal{J},\{P\}, \varphi(\pi)}$ has two objects and a single morphism between each pair of objects. The colimit over such category is the quotient

$$
\left(M\left(P, V_{\pi_{0}}, 0\right) \oplus M\left(P^{w_{0}}, V_{\pi_{0}^{w_{0}}}, 0\right)\right) / W
$$

of the direct sum of the two isomorphic $\left(\mathfrak{g}, K_{\infty} ; G\left(\mathbb{A}_{f}\right)\right)$-modules by the $\left(\mathfrak{g}, K_{\infty} ; G\left(\mathbb{A}_{f}\right)\right)$-submodule, again denoted $W$, consisting of all $x-M\left(w_{0}\right) x$, where $x \in M\left(P, V_{\pi_{0}}, 0\right)$. However, this quotient is clearly isomorphic to one of the two isomorphic summands. Hence,

$$
\mathcal{A}_{\mathcal{J},\{P\}, \varphi(\pi)}^{0} \cong I\left(0, V_{\pi_{0}}\right) \otimes S\left(\check{\mathfrak{a}}_{P, \mathbb{C}}^{G}\right)
$$

in this case.

REMARK 3.2. The definition of the intertwining operator $M\left(w_{0}\right)$ in $[7$, page 234] involves not only the standard intertwining operators on the induced representations, but also their derivatives. Since these derivatives are no longer intertwining operators, the 1-eigenspace

$$
\left(I\left(0, V_{\pi_{0}}\right) \otimes S\left(\check{\mathfrak{a}}_{P, \mathbb{C}}^{G}\right)\right)^{+}
$$

of $M\left(w_{0}\right)$ appearing in Theorem 3.1 is not easy to describe as a $\left(\mathfrak{g}, K_{\infty} ; G\left(\mathbb{A}_{f}\right)\right)$ module. However, one can show that as a vector space it is isomorphic to the direct sum

$$
\bigoplus_{j=0}^{\infty}\left(I\left(0, V_{\pi_{0}}\right)^{\epsilon_{j}} \otimes S_{j}\left(\check{\mathfrak{a}}_{P, \mathbb{C}}^{G}\right)\right),
$$

where $\epsilon_{j}=(-1)^{j}$, and $I\left(0, V_{\pi_{0}}\right)^{\epsilon_{j}}$ is the $\epsilon_{j}$-eigenspace of the action of the standard self-intertwining operator $M\left(0, w_{0}\right)$ acting on $I\left(0, V_{\pi_{0}}\right)$ as defined in [18, Sect. II.1.6], and $S_{j}\left(\check{\mathfrak{a}}_{P, \mathbb{C}}^{G}\right)$ is the $j$-th summand in the standard grading of the symmetric algebra.

We end this section with a corollary, in which we state separately the non-self-associate case, that is, $P^{w_{0}} \neq P$ or $\pi_{0}^{w_{0}} \neq \pi_{0}$.

COROLlary 3.3. Let $G$ be a connected reductive linear algebraic group defined over $\mathbb{Q}$. Let $P$ be a standard maximal proper parabolic $\mathbb{Q}$-subgroup of $G$, and $\pi$ a cuspidal automorphic representation of $L_{P}(\mathbb{A})$. Suppose that one of the following conditions hold:

- either $P$ is not self-associate, that is, $P^{w_{0}} \neq P$,

- or $P$ is self-associate, but $\pi_{0}^{w_{0}} \neq \pi_{0}$.

Then, the Franke filtration of the $\left(\mathfrak{g}, K_{\infty} ; G\left(\mathbb{A}_{f}\right)\right)$-module $\mathcal{A}_{\mathcal{J},\{P\}, \varphi(\pi)}$ is onestep filtration, and

$$
\mathcal{A}_{\mathcal{J},\{P\}, \varphi(\pi)} \cong I\left(s_{0} \tilde{\alpha}, V_{\pi_{0}}\right) \otimes S\left(\check{\mathfrak{a}}_{P, \mathbb{C}}^{G}\right),
$$

as a $\left(\mathfrak{g}, K_{\infty} ; G\left(\mathbb{A}_{f}\right)\right)$-module. 
Proof. According to [18, Sect. IV.3], if any of the two conditions in the corollary is satisfied, the Eisenstein series attached to $\pi_{0}$ have no poles in the right half-plane $\operatorname{Re}(s) \geq 0$. Hence, in terminology of the proof of Theorem 3.1 , we are either in case 2 , or in case 4 . In both cases, by Theorem 3.1, the Franke filtration is one-step filtration, and $\mathcal{A}_{\mathcal{J},\{P\}, \varphi(\pi)}$ is as stated in the corollary.

\section{Corollaries for $\mathbb{Q}$-SPlit Classical groups}

For $\mathbb{Q}$-split classical groups, in some cases it is possible to make the statement of Theorem 3.1 even more precise. This is due to the fact that the required analytic behavior of the Eisenstein series is known. We retain the notation of Section 3.

We begin with the case of the general linear group over $\mathbb{Q}$. The description of the lower filtration step in this case was already used in Section 5 of [8]. The analytic properties of the Eisenstein series are obtained in [17]. In this section we write $\pi_{0}$ instead of $V_{\pi_{0}}$ for the $\pi_{0}$-isotypic space of cuspidal automorphic forms on $G L_{n}(\mathbb{A})$. This is to stress that, by the multiplicity one for $G L_{n}(\mathbb{A})$ (cf. [22, Thm. 5.5]), $V_{\pi_{0}}$ is isomorphic to $\pi_{0}$.

Corollary 4.1. Let $G=G L_{n}$ be the general linear group of $n \times n$ invertible matrices over $\mathbb{Q}$. Let $P$ be a standard maximal proper parabolic $\mathbb{Q}$ subgroup of $G$. Then $L_{P} \cong G L_{m} \times G L_{m^{\prime}}$ for some positive integers $m, m^{\prime} \in$ $\mathbb{Z}_{>0}$ such that $m+m^{\prime}=n$, and

$$
\tilde{\alpha}=\left(\operatorname{det}_{G L_{m}}\right)^{m^{\prime} / n} \otimes\left(\operatorname{det}_{G L_{m^{\prime}}}\right)^{-m / n} \in \check{\mathfrak{a}}_{P}^{G} .
$$

Let $\pi \otimes \pi^{\prime}$ be a cuspidal automorphic representation of $L_{P}(\mathbb{A})$, where $\pi$ and $\pi^{\prime}$ are cuspidal automorphic representations of $G L_{m}(\mathbb{A})$ and $G L_{m^{\prime}}(\mathbb{A})$, respectively. Writing

$$
\begin{aligned}
\pi \otimes \pi^{\prime} & \cong \pi_{0}\left|\operatorname{det}_{G L_{m}}\right|^{s_{0} m^{\prime} / n} \otimes \pi_{0}^{\prime}\left|\operatorname{det}_{G L_{m^{\prime}}}\right|^{-s_{0} m / n} \\
& =\left(\pi_{0} \otimes \pi_{0}^{\prime}\right) \otimes \exp \left\langle s_{0} \tilde{\alpha}, H_{P}(\cdot)\right\rangle
\end{aligned}
$$

where $\pi_{0}$ and $\pi_{0}^{\prime}$ are unitary cuspidal automorphic representations of $G L_{m}(\mathbb{A})$ and $G L_{m^{\prime}}(\mathbb{A})$, respectively, we may assume, as in Section 3, that $s_{0} \geq 0$.

(1) If the following conditions

- $n$ is even and $m=m^{\prime}=n / 2$,

- $\pi_{0} \cong \pi_{0}^{\prime}$ and $s_{0}=1$, i.e.,

$$
\pi \otimes \pi^{\prime} \cong \pi_{0}\left|\operatorname{det}_{G L_{n / 2}}\right|^{1 / 2} \otimes \pi_{0}\left|\operatorname{det}_{G L_{n / 2}}\right|^{-1 / 2},
$$

are satisfied, then the Franke filtration of the $\left(\mathfrak{g}, K_{\infty} ; G\left(\mathbb{A}_{f}\right)\right)$-module $\mathcal{A}_{\mathcal{J},\{P\}, \varphi(\pi)}$ is a two-step filtration

$$
\{0\} \subset \mathcal{L}_{\mathcal{J},\{P\}, \varphi(\pi)} \subset \mathcal{A}_{\mathcal{J},\{P\}, \varphi(\pi)} .
$$


The lower filtration step is isomorphic to

$$
\mathcal{L}_{\mathcal{J},\{P\}, \varphi(\pi)} \cong J\left(\tilde{\alpha}, \pi_{0} \otimes \pi_{0}\right)
$$

as a $\left(\mathfrak{g}, K_{\infty} ; G\left(\mathbb{A}_{f}\right)\right)$-module, where $J\left(\tilde{\alpha}, \pi_{0} \otimes \pi_{0}\right)$ is the unique irreducible quotient of the induced representation

$$
I\left(\tilde{\alpha}, \pi_{0} \otimes \pi_{0}\right)=\operatorname{Ind}_{P(\mathbb{A})}^{G L_{n}(\mathbb{A})}\left(\pi_{0}\left|\operatorname{det}_{n / 2}\right|^{1 / 2} \otimes \pi_{0}\left|\operatorname{det}_{n / 2}\right|^{-1 / 2}\right) .
$$

The quotient is isomorphic to

$$
\begin{aligned}
& \mathcal{A}_{\mathcal{J},\{P\}, \varphi(\pi)} / \mathcal{L}_{\mathcal{J},\{P\}, \varphi(\pi)} \\
& \quad \cong I\left(\tilde{\alpha}, \pi_{0} \otimes \pi_{0}\right) \otimes S\left(\check{\mathfrak{a}}_{P, \mathbb{C}}^{G}\right) \\
& \quad \cong \operatorname{Ind}_{P(\mathbb{A})}^{G L_{n}(\mathbb{A})}\left(\pi_{0}\left|\operatorname{det}_{n / 2}\right|^{1 / 2} \otimes \pi_{0}\left|\operatorname{det}_{n / 2}\right|^{-1 / 2}\right) \otimes S\left(\check{\mathfrak{a}}_{P, \mathbb{C}}^{G}\right)
\end{aligned}
$$

as a $\left(\mathfrak{g}, K_{\infty} ; G\left(\mathbb{A}_{f}\right)\right)$-module.

(2) If conditions in (1) are not satisfied, then the Franke filtration of the $\left(\mathfrak{g}, K_{\infty} ; G\left(\mathbb{A}_{f}\right)\right)$-module $\mathcal{A}_{\mathcal{J},\{P\}, \varphi(\pi)}$ is one-step filtration. In this case, (a) if

- $n$ is even and $m=m^{\prime}=n / 2$,

- $\pi_{0} \cong \pi_{0}^{\prime}$ and $s_{0}=0$, i.e., $\pi \otimes \pi^{\prime} \cong \pi_{0} \otimes \pi_{0}$,

then

$$
\begin{aligned}
\mathcal{A}_{\mathcal{J},\{P\}, \varphi(\pi)} & \cong\left(I\left(0, \pi_{0} \otimes \pi_{0}\right) \otimes S\left(\check{\mathfrak{a}}_{P, \mathbb{C}}^{G}\right)\right)^{+} \\
& \cong\left(\operatorname{Ind}_{P(\mathbb{A})}^{G L_{n}(\mathbb{A})}\left(\pi_{0} \otimes \pi_{0}\right) \otimes S\left(\check{\mathfrak{a}}_{P, \mathbb{C}}^{G}\right)\right)^{+}
\end{aligned}
$$

as $a\left(\mathfrak{g}, K_{\infty} ; G\left(\mathbb{A}_{f}\right)\right)$-module, where + in the exponent denotes the 1-eigenspace of the action of $M\left(w_{0}\right)$ as in Theorem 3.1,

(b) otherwise, that is, if neither the conditions in (1), nor those in (2a), are satisfied, then

$$
\begin{aligned}
& \mathcal{A}_{\mathcal{J},\{P\}, \varphi(\pi)} \cong I\left(s_{0} \tilde{\alpha}, \pi_{0} \otimes \pi_{0}^{\prime}\right) \otimes S\left(\check{\mathfrak{a}}_{P, \mathbb{C}}^{G}\right) \\
& \cong \operatorname{Ind}_{P(\mathbb{A})}^{G L_{n}(\mathbb{A})}\left(\pi_{0}\left|\operatorname{det}_{G L_{m}}\right|^{s_{0} m^{\prime} / n} \otimes \pi_{0}^{\prime}\left|\operatorname{det}_{G L_{m^{\prime}}}\right|^{-s_{0} m / n}\right) \otimes S\left(\check{\mathfrak{a}}_{P, \mathbb{C}}^{G}\right) \\
& \text { as a }\left(\mathfrak{g}, K_{\infty} ; G\left(\mathbb{A}_{f}\right)\right) \text {-module. }
\end{aligned}
$$

Proof. In this case the analytic behavior of the Eisenstein series is known from [17]. The conditions in part (1) of the corollary are precisely the conditions for the Eisenstein series attached to $\pi_{0} \otimes \pi_{0}^{\prime}$ to have a pole inside $\operatorname{Re}(s) \geq 0$. The description of the space of residues as the unique irreducible quotient of an induced representation is also due to [17], and, according to Theorem 3.1, this space is isomorphic to the lower filtration step in the Franke filtration. The description of the quotient in part (1) follows then directly from Theorem 3.1.

In part (2) of the corollary, the Eisenstein series has no pole inside $\operatorname{Re}(s) \geq$ 0 (again by [17]). Hence, by Theorem 3.1, the Franke filtration of $\mathcal{A}_{\mathcal{J},\{P\}, \varphi(\pi)}$ 
is one step filtration. The description of $\mathcal{A}_{\mathcal{J},\{P\}, \varphi(\pi)}$ follows directly from Theorem 3.1. Observe that part (2a) of the corollary is precisely the case $s_{0}=0, P^{w_{0}}=P$ and $\left(\pi_{0} \otimes \pi_{0}^{\prime}\right)^{w_{0}} \cong \pi_{0} \otimes \pi_{0}^{\prime}$, in which the 1-eigenspace of the intertwining operator $M\left(w_{0}\right)$ appears in Theorem 3.1.

Finally, we consider the case of the $\mathbb{Q}$-split symplectic or special orthogonal group $G$ of $\mathbb{Q}$-rank $n \geq 2$, and the associate class of the Siegel standard maximal proper parabolic $\mathbb{Q}$-subgroup of $G$. This is the parabolic $\mathbb{Q}$-subgroup $P$ of $G$ such that the unique simple root $\alpha$ not being the root of the Levi factor $L_{P}$ is the last simple root of $G$ in the standard ordering (as in [5]). The Levi factor $L_{P}$ of $P$ is isomorphic to $G L_{n}$.

In this case the analytic properties of the Eisenstein series are studied in [10], using the results of [6]. The poles inside $R e(s)>0$ are determined in terms of the poles of the symmetric or exterior square $L$-functions (and for the symplectic group also the non-vanishing of principal $L$-functions for $\left.G L_{n}\right)$. The paper [10] argues further, assuming Arthur's description of the discrete spectrum for classical groups (cf. [1], [2]), conjectural at the time, that the only possible pole inside $\operatorname{Re}(s)>0$ of the $L$-functions in question is at $s=1$.

Corollary 4.2. Let $G$ be one of the $\mathbb{Q}$-split classical groups $S p_{2 n}$, $S O_{2 n+1}$, and $S O_{2 n}$ of $\mathbb{Q}$-rank $n \geq 2$. Let $P$ be the Siegel standard maximal proper parabolic $\mathbb{Q}$-subgroup of $G$, i.e., the standard parabolic $\mathbb{Q}$-subgroup such that the unique simple root $\alpha$ of $G$ that is not a root of $L_{P}$ is the last root in the standard ordering of simple roots of $G$ (as in [5]). Then $L_{P} \cong G L_{n}$, and

Let

$$
\tilde{\alpha}= \begin{cases}\operatorname{det}, & \text { for } G=S p_{2 n}, \\ \operatorname{det}^{1 / 2}, & \text { for } G=S O_{2 n} \text { and } G=S O_{2 n+1} .\end{cases}
$$

$\pi \cong \pi_{0}|\operatorname{det}|^{s_{0}} \cong\left\{\begin{array}{l}\pi_{0} \otimes \exp \left\langle s_{0} \tilde{\alpha}, H_{P}(\cdot)\right\rangle, \text { for } G=S p_{2 n}, \\ \pi_{0} \otimes \exp \left\langle 2 s_{0} \tilde{\alpha}, H_{P}(\cdot)\right\rangle, \text { for } G=S O_{2 n+1} \text { and } G=S O_{2 n},\end{array}\right.$

be a cuspidal automorphic representation of $L_{P}(\mathbb{A})$, where $\pi_{0}$ is unitary cuspidal, and $s_{0} \geq 0$. Note that we use, as in [21, page 5], a slightly different normalization of $s_{0}$ in the case of special orthogonal groups. Assume Arthur's description of the discrete spectrum for classical groups (cf. [1], [2]).

(1) Suppose that the following conditions

- $\pi_{0}$ is self-contragredient,

$$
\text { - } \begin{cases}\text { if } G=S O_{2 n+1}, \text { then } & \pi_{0} \text { is orthogonal, i.e., } \\ & L\left(s, \pi_{0}, S y m^{2}\right) \\ & \text { has a pole at } s=1, \\ & \pi_{0} \text { is symplectic, i.e., } \\ \text { if } G=S p_{2 n} \text { or } G=S O_{2 n}, \text { then } \quad & L\left(s, \pi_{0}, \wedge^{2}\right) \\ & \text { has a pole at } s=1,\end{cases}
$$


- if $G=S p_{2 n}$, then $L\left(1 / 2, \pi_{0}\right) \neq 0$,

- $s_{0}=1 / 2$,

are satisfied, where $L\left(s, \pi_{0}\right)$ is the principal $L$-function, and $L\left(s, \pi_{0}\right.$, $\left.S_{y m^{2}}\right)$ (resp. $\left.L\left(s, \pi_{0}, \wedge^{2}\right)\right)$ is the symmetric (resp. exterior) square $L$ function. Then, the Franke filtration of the $\left(\mathfrak{g}, K_{\infty} ; G\left(\mathbb{A}_{f}\right)\right)$-module $\mathcal{A}_{\mathcal{J},\{P\}, \varphi(\pi)}$ is a two-step filtration

$$
\{0\} \subset \mathcal{L}_{\mathcal{J},\{P\}, \varphi(\pi)} \subset \mathcal{A}_{\mathcal{J},\{P\}, \varphi(\pi)} .
$$

The lower filtration step is isomorphic to

$$
\mathcal{L}_{\mathcal{J},\{P\}, \varphi(\pi)} \cong \begin{cases}J\left((1 / 2) \tilde{\alpha}, \pi_{0}\right), & \text { for } G=S p_{2 n}, \\ J\left(\tilde{\alpha}, \pi_{0}\right), & \text { for } G=S O_{2 n+1} \text { and } G=S O_{2 n},\end{cases}
$$

as a $\left(\mathfrak{g}, K_{\infty} ; G\left(\mathbb{A}_{f}\right)\right)$-module, where the right-hand side denotes the unique irreducible quotient of the induced representation

$\operatorname{Ind}_{P(\mathbb{A})}^{G(\mathbb{A})}\left(\pi_{0}|\operatorname{det}|^{1 / 2}\right) \cong \begin{cases}I\left((1 / 2) \tilde{\alpha}, \pi_{0}\right), & \text { for } G=S p_{2 n}, \\ I\left(\tilde{\alpha}, \pi_{0}\right), & \text { for } G=S O_{2 n+1} \text { and } G=S O_{2 n} .\end{cases}$

The quotient is isomorphic to

$$
\begin{aligned}
& \mathcal{A}_{\mathcal{J},\{P\}, \varphi(\pi)} / \mathcal{L}_{\mathcal{J},\{P\}, \varphi(\pi)} \cong \begin{cases}I\left((1 / 2) \tilde{\alpha}, \pi_{0}\right) \otimes S\left(\check{\mathfrak{a}}_{P, \mathbb{C}}\right), & \text { for } G=S p_{2 n} \\
I\left(\tilde{\alpha}, \pi_{0}\right) \otimes S\left(\check{\mathfrak{a}}_{P, \mathbb{C}}\right), & \text { for } G=S O_{2 n+1}\end{cases} \\
& \cong \operatorname{Ind}_{P(\mathbb{A})}^{G(\mathbb{A})}\left(\pi_{0}|\operatorname{det}|^{1 / 2}\right) \otimes S\left(\check{\mathfrak{a}}_{P, \mathbb{C}}\right)
\end{aligned}
$$

as a $\left(\mathfrak{g}, K_{\infty} ; G\left(\mathbb{A}_{f}\right)\right)$-module.

(2) If the conditions in (1) are not satisfied, then the Franke filtration of the $\left(\mathfrak{g}, K_{\infty} ; G\left(\mathbb{A}_{f}\right)\right)$-module $\mathcal{A}_{\mathcal{J},\{P\}, \varphi(\pi)}$ is one-step filtration. In this case:

(a) if

- $\pi_{0}$ is self-contragredient,

- if $n$ is odd, then $G \neq S O_{2 n}$,

- $s_{0}=0$,

then

$$
\mathcal{A}_{\mathcal{J},\{P\}, \varphi(\pi)} \cong\left(I\left(0, \pi_{0}\right) \otimes S\left(\check{\mathfrak{a}}_{P, \mathbb{C}}\right)\right)^{+} \cong\left(\operatorname{Ind}_{P(\mathbb{A})}^{G(\mathbb{A})}\left(\pi_{0}\right) \otimes S\left(\check{\mathfrak{a}}_{P, \mathbb{C}}\right)\right)^{+}
$$

as a $\left(\mathfrak{g}, K_{\infty} ; G\left(\mathbb{A}_{f}\right)\right)$-module, where + in the exponent denotes the 1-eigenspace of the intertwining operator $M\left(w_{0}\right)$ as in Theorem 3.1,

(b) otherwise, that is, if neither conditions in (1), nor conditions in (2a), are satisfied, then

$$
\begin{aligned}
\mathcal{A}_{\mathcal{J},\{P\}, \varphi(\pi)} \cong\left\{\begin{array}{l}
I\left(s_{0} \tilde{\alpha}, \pi_{0}\right) \otimes S\left(\check{\mathfrak{a}}_{P, \mathbb{C}}\right), \quad \text { for } G=S p_{2 n} \\
I\left(2 s_{0} \tilde{\alpha}, \pi_{0}\right) \otimes S\left(\check{\mathfrak{a}}_{P, \mathbb{C}}\right), \text { for } G=S O_{2 n+1} \text { and } G=S O_{2 n}
\end{array}\right. \\
\cong \operatorname{Ind}_{P(\mathbb{A})}^{G(\mathbb{A})}\left(\pi_{0}|\operatorname{det}|^{s_{0}}\right) \otimes S\left(\check{\mathfrak{a}}_{P, \mathbb{C}}\right)
\end{aligned}
$$


as $a\left(\mathfrak{g}, K_{\infty} ; G\left(\mathbb{A}_{f}\right)\right)$-module.

Proof. Assuming Arthur's description of the discrete spectrum of $G(\mathbb{A})$ (cf. [1, Sect. 30], [2]), and relying on the results of [6], the analytic behavior for $\operatorname{Re}(s) \geq 0$ of the Eisenstein series attached to $\pi_{0}$ is determined in [10]. There is a pole inside $R e(s) \geq 0$ if and only if the conditions in part (1) of the corollary are satisfied. Hence, according to Theorem 3.1, the Franke filtration is a two-step filtration, and the lower filtration step is spanned by the residues of the Eisenstein series.

The space of residues is described in [10] as the image of the normalized intertwining operator (as defined in [20]) that intertwines

$$
\operatorname{Ind}_{P(\mathbb{A})}^{G(\mathbb{A})}\left(\pi_{0}|\operatorname{det}|^{1 / 2}\right) \rightarrow \operatorname{Ind}_{P(\mathbb{A})}^{G(\mathbb{A})}\left(\pi_{0}|\operatorname{det}|^{-1 / 2}\right) .
$$

However, Lemma 4.4 shows that the image of that operator is irreducible, and isomorphic to the unique irreducible quotient denoted by $J$ in the corollary.

The description of the quotient in part (1), as well as part (2) of the corollary follow directly from Theorem 3.1. For part (2a) observe that $\pi_{0}$ is self-contragredient if and only if $\pi_{0}^{w_{0}} \cong \pi_{0}$, and that the Siegel parabolic $\mathbb{Q}$-subgroup of $G$ is self-associate, except if $n$ is odd and $G=S O_{2 n}$ (cf. [21, Lemma 3.4]).

REMark 4.3. If $n$ is odd, and either $G=S p_{2 n}$, or $G=S O_{2 n}$, then the Franke filtration of $\mathcal{A}_{\mathcal{J},\{P\}, \varphi(\pi)}$ is one-step filtration for any $\pi$. This follows from the fact that there are no symplectic cuspidal automorphic representations of $G L_{n}(\mathbb{A})$ with $n$ odd (cf. [1, Sect. 30]), and thus, the second condition in part (1) of the corollary is never satisfied.

LEMMA 4.4. Let $G$ be one of the $\mathbb{Q}$-split classical groups $S p_{2 n}, S O_{2 n+1}$ and $S O_{2 n}$ of $\mathbb{Q}$-rank $n \geq 2$. Let $P$ be the Siegel parabolic $\mathbb{Q}$-subgroup of $G$ as in Corollary 4.2. Let $\pi_{0}$ be a unitary cuspidal automorphic representation of $L_{P}(\mathbb{A})$. Then, the image of the normalized intertwining operator

$$
\operatorname{Ind}_{P(\mathbb{A})}^{G(\mathbb{A})}\left(\pi_{0}|\operatorname{det}|^{1 / 2}\right) \rightarrow \operatorname{Ind}_{P(\mathbb{A})}^{G(\mathbb{A})}\left(\pi_{0}|\operatorname{det}|^{-1 / 2}\right)
$$

as defined in [20], is irreducible, and isomorphic to the unique irreducible quotient of

$$
\operatorname{Ind}_{P(\mathbb{A})}^{G(\mathbb{A})}\left(\pi_{0}|\operatorname{det}|^{1 / 2}\right) \text {. }
$$

Proof. The lemma follows from the classification of the generic unitary dual of $G L_{n}\left(\mathbb{Q}_{v}\right)$, which is obtained by Tadic in [23] for a finite place $v$, and by Vogan in [24] for $v=\infty$. In both cases, since $\pi_{0, v}$ is the local component at $v$ of a unitary cuspidal automorphic representation, it is unitary and generic. Hence, by the classification mentioned above, it is a fully induced representation to $L_{P}\left(\mathbb{Q}_{v}\right) \cong G L_{n}\left(\mathbb{Q}_{v}\right)$ from a tensor product of the form

$$
\delta_{1}|\operatorname{det}|^{e_{1}} \otimes \cdots \otimes \delta_{l}|\operatorname{det}|^{e_{l}}
$$


on the Levi factor of the appropriate parabolic $\mathbb{Q}$-subgroup of $L_{P} \cong G L_{n}$, where $\delta_{i}$ are unitary square-integrable representations of the general linear groups, and $\left|e_{i}\right|<1 / 2$, for $i=1, \ldots, l$. We may assume that $e_{1} \geq \cdots \geq e_{l}$, because the induced representation is irreducible. By the standard argument, relying on induction in stages, the image of the intertwining operator above is isomorphic to the image of the longest intertwining operator acting on the induced representation

$$
\operatorname{Ind}_{Q\left(\mathbb{Q}_{v}\right)}^{G\left(\mathbb{Q}_{v}\right)}\left(\delta_{1}|\operatorname{det}|^{e_{1}+1 / 2} \otimes \cdots \otimes \delta_{l}|\operatorname{det}|^{e_{l}+1 / 2}\right),
$$

for the appropriate parabolic $\mathbb{Q}$-subgroup $Q$ of $G$. But this is a standard module for $G\left(\mathbb{Q}_{v}\right)$, as

$$
e_{1}+1 / 2 \geq \cdots \geq e_{l}+1 / 2>0,
$$

so that the image is irreducible at every place by the Langlands classification (cf. [4]).

REMARK 4.5. We finish with a remark on the 1-eigenspaces appearing in the corollaries. As already mentioned in Remark 3.2, these eigenspaces are not easy to describe as $\left(\mathfrak{g}, K_{\infty} ; G\left(\mathbb{A}_{f}\right)\right)$-modules, since $M\left(w_{0}\right)$ is defined in terms of standard intertwining operators and their derivatives. However, as a vector space, it can be described in terms of \pm 1 -eigenspaces of the standard intertwining operator $M\left(0, w_{0}\right)$ acting on the induced representation $I\left(0, \pi_{0} \otimes\right.$ $\left.\pi_{0}\right)$ for $G=G L_{n}$, and $I\left(0, \pi_{0}\right)$ for $G$ one of the $\mathbb{Q}$-split classical groups. Using the Langlands-Shahidi method to normalize standard intertwining operators (cf. [20]), and passing to the local intertwining operators, one can show by the theory of $R$ groups (cf. [9]) that $M\left(0, w_{0}\right)$ is not a scalar operator whenever the induced representation is reducible. If the induced representation is irreducible, one can write the sign of $M\left(0, w_{0}\right)$ in terms of $L$-functions using local coefficients of Shahidi (cf. [19], [20]).

\section{REFERENCES}

[1] J. Arthur, An introduction to the trace formula, in: Harmonic analysis, the trace formula, and Shimura varieties (J. Arthur, D. Ellwood, R. Kottwitz, eds.), Clay Math. Proc. 4, Amer. Math. Soc., Providence, 2005, 1-263.

[2] J. Arthur, The endoscopic classification of representations: orthogonal and symplectic groups, preprint, http://www.claymath.org/cw/arthur/pdf/Book.pdf.

[3] A. Borel and H. Jacquet, Automorphic forms and automorphic representations, in: Automorphic forms, representations and $L$-functions (A. Borel, W. Casselman, eds.), Proc. Sympos. Pure Math. XXXIII Part 1, Amer. Math. Soc., Providence, 1979, 189-207.

[4] A. Borel and N. Wallach, Continuous cohomology, discrete subgroups, and representations of reductive groups, Second edition, Amer. Math. Soc., Providence, 2000.

[5] N. Bourbaki, Groupes et algèbres de Lie, Chapitre IV,V,VI, Hermann, Paris, 1968.

[6] J. W. Cogdell, H. H. Kim, I. I. Piatetski-Shapiro and F. Shahidi, Functoriality for the classical groups, Publ. Math. Inst. Hautes Études Sci. 99 (2004), 163-233. 
[7] J. Franke, Harmonic analysis in weighted $L_{2}$-spaces, Ann. Sci. École Norm. Sup. (4) 31 (1998), 181-279.

[8] J. Franke and J. Schwermer, A decomposition of spaces of automorphic forms, and the Eisenstein cohomology of arithmetic groups, Math. Ann. 311 (1998), 765-790.

[9] D. Goldberg, Reducibility of induced representations for $S p(2 n)$ and $S O(n)$, Amer. J. Math. 116 (1994), 1101-1151.

[10] N. Grbac, On the residual spectrum of split classical groups supported in the Siegel maximal parabolic subgroup, Monatsh. Math. 163 (2011), 301-314.

[11] N. Grbac and H. Grobner, The residual Eisenstein cohomology of Sp 4 over a totally real number field, to appear in Trans. Amer. Math. Soc.

[12] N. Grbac and J. Schwermer, On residual cohomology classes attached to relative rank one Eisenstein series for the symplectic group, Int. Math. Res. Not. IMRN 2011, 1654-1705.

[13] N. Grbac and J. Schwermer, An exercise in automorphic cohomology - the case $G L_{2}$ over a quaternion algebra, in: Arithmetic Geometry and Automorphic Forms (J. Funke, J. Cogdell, M. Rapoport, T. Yang, eds.), Adv. Lect. Math. (ALM) 19, Higher Education Press and International Press, Beijing-Boston, 2011, 209-252.

[14] R. P. Langlands, Letter to A. Borel, dated October 25, 1972.

[15] R. P. Langlands, On the functional equations satisfied by Eisenstein series, SpringerVerlag, Berlin-New York, 1976.

[16] S. MacLane, Categories for the working mathematician, Springer-Verlag, New YorkBerlin, 1971.

[17] C. Mœglin and J.-L. Waldspurger, Le spectre résiduel de $G L(n)$, Ann. Sci. École Norm. Sup. (4) 22 (1989), 605-674.

[18] C. Mœglin and J.-L. Waldspurger, Spectral decomposition and Eisenstein series, Cambridge University Press, Cambridge, 1995.

[19] F. Shahidi, On certain L-functions, Amer. J. Math. 103 (1981), 297-355.

[20] F. Shahidi, A proof of Langlands' conjecture on Plancherel measures; complementary series for p-adic groups, Ann. of Math. (2) 132 (1990), 273-330.

[21] F. Shahidi, Twisted endoscopy and reducibility of induced representations for $p$-adic groups, Duke Math. J. 66 (1992), 1-41.

[22] J. A. Shalika, The multiplicity one theorem for $G L_{n}$, Ann. of Math. (2) 100 (1974), 171-193.

[23] M. Tadić, Classification of unitary representations in irreducible representations of general linear group (non-Archimedean case), Ann. Sci. École Norm. Sup. (4) 19 (1986), 335-382.

[24] D. A. Vogan Jr., The unitary dual of $G L(n)$ over an Archimedean field, Invent. Math. 83 (1986), 449-505.

\section{N. Grbac}

Department of Mathematics

University of Rijeka

Radmile Matejčić 2, HR-51000 Rijeka

Croatia

E-mail: neven.grbac@math.uniri.hr

Received: 10.12.2011. 